\title{
Prediction of Roughness Heights of Milled Surfaces for Product Quality Prediction and Tool Condition Monitoring
}

\author{
J.J. Ng${ }^{1}$, Z.W. Zhong, ${ }^{1, *}$ T.I. Liu ${ }^{2,3}$ \\ 1. School of Mechanical and Aerospace Engineering, Nanyang Technological University, 50 Nanyang Avenue, \\ 639798, Singapore \\ 2. Department of Mechanical Engineering, California State University, Sacramento, California 95819, USA \\ 3. Institute of Manufacturing Technology, National Taipei University of Technology, 10608, Taiwan \\ E-mail address: mzwzhong@ntu.edu.sg (Corresponding author)
}

Received: 5 September 2019; Accepted: 27 September 2019; Available online:15 November 2019

\begin{abstract}
The objective of this research is to predict the roughness heights of milled surfaces, which indicates product quality and tool conditions. Two experiments are carried out to evaluate relevant factors such as vibration, force, and surface roughness. The purpose of the first experiment is to find out the limits of the machining variables compared to the constraints of the materials. The purpose of the second experiment is to identify, collect, and compare how each factor affects product quality and tool conditions. Based on this study, the vibration, force, and surface roughness are good indicators for tool conditions. When the magnitudes of the vibration and force increase, the surface roughness also increases. The increase in surface roughness with constant cutting parameters indicates the degrading of product quality and the decrease of the tool life. Thus, the variables, such as vibration and forces, are used as the inputs, and the surface roughness is used as the output of neural networks. By optimizing the network variables, it has been found that a 4,4,8,1 neural network can achieve the least absolute error, and accurately predict the actual roughness heights collected from the experiment. The minimum error of the prediction of surface roughness is $0.11 \%$, the average error is $2.11 \%$, and the maximum error is $6.98 \%$. The prediction of surface roughness of milled surfaces is very important for the product quality prediction and tool condition monitoring.
\end{abstract}

Keywords: Roughness heights; Milled surfaces; Product quality; Tool conditions; Vibration; Force.

\section{Introduction}

In recent years, there is a need to identify product quality and tool conditions timely, so as to maximize the reliability of the tool and optimize the output of the components to achieve high efficiency and a low rejection rate $[1]$.

The advancement of the technology in monitoring sensors allows signal processing, data collection, optimization, and control to be possible, which provides close analyses capable of machine tool diagnostics and in-process quality control [2]. These monitoring systems are used to improve machining quality and reliability for the design and development of new machine tools. Such monitoring systems may integrate artificial neural networks, which have been used more often and widely in designing models for different machining processes, because of their capability in learning and generalization. These neural networks are also able to accommodate variables from machining processes, which are complex or non-linear, to adapt to the changing environment and provide resistance to missing information. Some of the neural networks are already in place for machining operations and have proven their effectiveness in optimizing machining processes.

This research focuses on identifying factors that will contribute to product quality prediction and tool condition monitoring, and analyzing correlations between these factors. It creates a neural network to train and learn the relationship between various factors and to optimize the network, so as to predict product quality and tool status. The combination of the cutting tool material and workpiece material is widely used in the industry. However, research on intelligent manufacturing is still lacking. This research utilizes an innovative approach for the prediction of surface roughness of milled surfaces, which would contribute significantly to engineering, particularly to the field of intelligent manufacturing.

\section{Literature review: Tool Condition Monitoring (TCM) systems}


A TCM system is a fundamental requirement for the control of a machining process. It consists of different kinds of sensors for data collection, and the collected information is used for analyses [3]. The ability for TCM to detect and identify the tool failure is critical for enhancing the productivity in metal cutting. In other words, the TCM system achieves high machining accuracy, enhances production efficiency, reduces machine downtime and tooling costs, targets optimal utilization of machine tools, and lowers the costs for more reliable unmanned automated manufacturing systems [4].

\subsection{Direct and indirect TCM techniques}

Direct techniques sense tool conditions by performing analyses on the measurements of the tools directly by interrupting the machining processes. Direct techniques include optical measurements, visual inspection, usage of profilometers, usage of radioactive and electrical resistance sensors, and measurements of chip sizes [5]. The main disadvantage of such direct systems is the failure to detect any significant deterioration that occurs in between measurements. Direct techniques also result in an increase of machine downtime and the cost for the TCM system [6].

Indirect techniques, on the other hand, sense tool conditions by using secondary effects of a cutting process, such as the acoustic emission (AE), spindle/feed current, cutting forces, sound, and vibrations. Indirect sensors include, but are not limited to, cutting force dynamometers, AE sensors, and accelerometers, which are used to collect signals in real time for analyses. The main disadvantage of such systems is that the signals collected do not contain direct measurements of the tool conditions, and thus require additional systems to correlate the direct measurements with the tool conditions using techniques, such as regression analyses, neural networks, fuzzy logic, and neuro-fuzzy systems [7-11]. Indirect techniques also tend to be weakened by noise factors [12].

\subsection{General considerations}

Each of various advanced materials poses challenges in milling. Many companies often utilize high-speed-steel and carbide milling tools to achieve high quality via TCM, because producing high quality products is of much higher priority than the cost and the productivity [13-15]. Furthermore, knowing the tool life precisely will also allow the manufacturers to replace tools, well before the tools are completely worn-out. Thus, the advance in TCM will also help to ensure the manufacturers to improve machining efficiency and to reduce production costs.

\subsection{Artificial Neural Networks (ANNs)}

An ANN is a computer program simulating the human's brain and performing data processing. It is also used for neural computing and pattern recognition. It learns from training data with outputs and inputs. Based upon the training process, the output vector can be estimated for a given input vector. ANNs are very suitable to carry out the kinds of tasks that do not have perfect answers. They have many advantages such as computing very quickly, being a nonlinear transfer function to correlate the inputs and the outputs, and having various possible structures. ANNs can be applied to deduce the relationship between parameters obtained from observations. ANNs have been utilized for tool condition monitoring and predicting of tool wear and lives [16]. According to the researches, the multi-layer perception and the back propagation are widely used [17]. It has also been reported that using the networks can help to predict surface roughness of machined surfaces [18] and to explore the relationship between tool wear and cutting forces for various cutting conditions [15-17, 19]. It can also help to suggest cutting parameters for required turning processes [20].

\subsection{Multi-layer perception and back propagation algorithm}

The multi-layer perception is known as a multi-layer feed-forward ANN. It has been reported that the multilayer perception network has integrated signals like force ratio and machining speed, as the input variables to monitor flank wear on the cutting tool [21].

The back propagation algorithm calculates the error to adjust the weights to get the minimum error. An back propagation model has been applied to the prediction of the tool flank wear and crater of the tool [22]. It is reported that training conditions such as the initial weights, number of hidden neurons, and mean squared error can influence the network performance.

\section{Milling experiments}

\subsection{Outline of milling experiments}

The Kennametal insert grade KC275M was used for the milling experiments. It has a multi-layer physical vapor deposition (PVD) coating with a tough cobalt substrate of $11.5 \%$. It is highly recommended for milling workpieces made of materials like steel, stainless steel, and ductile cast iron. It can also perform well in machining hardened 
materials because of its good resistance to thermal shock, and because of its suitability for both dry and wet machining applications [23].

The AISI 1045 steel used in our experiments had to be machined to a dimension of $100 \mathrm{~mm}$ in width by 150 $\mathrm{mm}$ in length before a dynamometer could be mounted. The characteristics of the AISI 1045 steel offer good strength, toughness and wear resistance. It was also hardened and tempered to achieve a hardness of 30 Rockwell Hardness on the $\mathrm{C}$ scale (HRC) for experimental purposes, so as to achieve a faster rate for the tool to degrade. All hardness would be verified using a Rockwell hardness tester [23].

Tool wear and damage were classified according to the wear scale and how it progressed. Wear is the loss of material on a smaller scale and usually progresses continuously, while damage is on a larger scale than wear and occurs suddenly. Tool wear is described as the gradual failure of cutting tools due to regular machining operations. Tool condition monitoring is widely researched [15, 24].

Two experiments were conducted in this work. Experiment 1 was carried out to investigate the worst case scenario. The factors that were taken into consideration included the hardness of the material and milling parameters. The adjustments would be made on the milling parameters, which were confined by the allowable ranges due to the specifications of the Mazak FJV milling machine for safe operations. It was also important to obtain a worst case scenario to shorten the experimental time and to achieve reliable results.

Experiment 2 was conducted to analyze the effects of force and vibration on the tool life of carbide inserts during milling of AISI 1045 Hardened Tempered Steel. The milling parameters used were cutting speed = 140 $\mathrm{m} / \mathrm{min}$, feed rate $=1 \mathrm{~mm} / \mathrm{rev}$, and depth of cut $=1 \mathrm{~mm}$. The surface roughness was also recorded as it was closely related to the tool life. This was because the surface roughness increased as the tool began to degrade. The measurement values of feed, radial and axial forces and the vibration in the feed direction were collected from the experiment, because these measurements were expected to increase when a tool started to become dull or reach the end of the tool life.

\subsection{Effect of cutting forces on the tool condition}

The forces of the cutter tool acting on the workpiece during the milling operation were measured and recorded. Table 1 shows the initial and ending values of the feed, radial, and axial forces at the start and end of the milling operation. It also shows that the feed force increased to $700 \mathrm{~N}$ by $325 \mathrm{~N}$ from $375 \mathrm{~N}$, the radial force increased to $1500 \mathrm{~N}$ by $1050 \mathrm{~N}$ from $450 \mathrm{~N}$, and the axial force increased to $1000 \mathrm{~N}$ by $400 \mathrm{~N}$ from $600 \mathrm{~N}$.

According to the experiment, the feed force, radial force, and axial force increased gradually throughout the milling operation and reached their maximum measurements at the end, compared to the initial measurements. This showed that as the tool degraded, the cutting tool required greater forces to remove the material from the machined part. Tool wear such as flank wear would reduce the tool's ability to cut the material.

Table 1. Ranges of feed, radial and axial forces collected from experiments

\begin{tabular}{lll}
\hline Forces & Initial $(\mathrm{N})$ & End $(\mathrm{N})$ \\
\hline Feed & 375 & 700 \\
Radial & 450 & 1500 \\
Axial & 600 & 1000 \\
\hline
\end{tabular}

\subsection{Effect of vibration on the tool condition}

The experimental results in Table 2 show that the range of the feed vibration magnitude was from $40.58 \mathrm{dBV}$ to $75.83 \mathrm{dBV}$. The results show that the magnitude of the feed vibration increased throughout the milling operation.

The vibration can also be used to indicate the tool condition, because it intensifies as the tool approaches the end of its life. The vibration in the feed direction can be correlated with the feed force measurement, because both results show that when the feed force increases, it also causes the feed vibration of the workpiece to increase.

Table 2. Range of feed vibration collected from experiments

\begin{tabular}{lll}
\hline Feed vibration & Initial $(|\mathrm{dBV}|)$ & End $(|\mathrm{dBV}|)$ \\
\hline Towards accelerometer & 40.58 & 75.83 \\
Away from accelerometer & 41.65 & 73.64 \\
\hline
\end{tabular}

\subsection{Effect of surface roughness on the tool condition}

The measurement results for surface roughness are displayed in Table 3 in the form of average roughness (Ra).

The results show that the surface roughness increases as the tool life decreases. This was proven true, because the milling parameters were constant while the only determining factor was the tool wear. The machined surface had significant feed marks, which was not acceptable because of the poor surface quality. When the feed marks started to appear during the milling operation, a further milling operation resulted in the unacceptable surface 
roughness $(\mathrm{Ra})$ of $1.74 \mu \mathrm{m}$. Although surface roughness can be affected by various milling parameters, when the milling parameters are fixed to be constant, increased surface roughness is a sign of increased tool wear. Therefore, the roughness can be used to determine the tool condition.

Table 3. Surface roughness collected from experiments

\begin{tabular}{ll}
\hline Milling operation & Ra $(\mu \mathrm{m})$ \\
\hline 1st & 0.48 \\
2nd & 0.52 \\
3rd & 1.54 \\
4th & 1.74 \\
\hline
\end{tabular}

\section{Artificial neural networks for prediction of surface roughness}

\subsection{Applications of neural networks to product quality prediction and tool condition monitoring}

After obtaining the relationship of how different factors affected product quality and tool conditions, these factors were selected to build the neural network. The back propagation algorithm was used, which employed the difference between the targeted value and the estimated output for the training purpose. The multilayer perception was used to identify the complex data that were not linearly separable.

The error obtained during the development of the neural network was closely monitored for the modification of parameters of the network architecture. The parameters could be the numbers of neurons and layers in the network, or the type of activation functions used for each layer. These parameters were adjusted with the weight and bias during training to learn the relationship. Lastly, the neural network was optimized to be able to predict surface roughness of milled surfaces, which indicated the product quality and tool conditions, with the cutting forces and vibrations being the inputs, as shown in Figure 1.

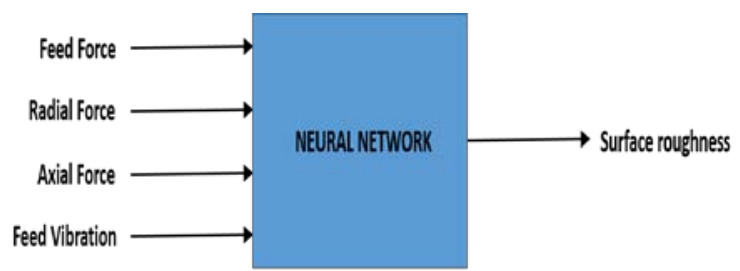

Figure 1. A simple sketch of the neuron network with 4 inputs and 1 output

\subsection{Effect of hidden neurons on training of neural networks}

All the data sets needed to be scaled to a range between -1 and 1 or 0 and -1 using the maximum and minimum values to compute. This was to ensure the network could be trained efficiently using either the hyperbolic tangent (tansig) or log sigmoid (logsig) function. By converting the magnitudes of the data sets to a common range, it reduced the complication of the weight or bias adjustment, and improved the training process. The number of hidden neurons in the hidden layers was calculated using the Wirdow rule [25] and Kolmogorov theorem [26].

The number of hidden neurons was found to be 3 and 9 using the Wirdow rule and Kolmogorov Theorem respectively. These values provided a range of the number of neurons that was used to generate the hidden layers. A number of neural networks were generated for the training and testing, as shown in Table 4.

During the trials, it was found that having the largest number of hidden neurons did not mean that the smallest percentage of the absolute error could be obtained. Therefore, the number of hidden neurons had to be tested along with different numbers of layers and activation functions, to achieve the best percentage of the absolute error.

Table 4. List of trial neural networks

\begin{tabular}{llll}
\hline Trial Network & 1 Hidden Layer & 2 Hidden layers & 3 Hidden layers \\
\hline 1 & $4,3,1$ & $4,3,6,1$ & $4,3,6,9,1$ \\
2 & $4,4,1$ & $4,4,8,1$ & $4,4,8,12,1$ \\
3 & $4,5,1$ & $4,5,10,1$ & $4,5,10,15,1$ \\
4 & $4,6,1$ & $4,6,12,1$ & $4,6,12,18,1$ \\
5 & $4,7,1$ & $4,7,14,1$ & $4,7,14,21,1$ \\
6 & $4,8,1$ & $4,8,16,1$ & $4,8,16,24,1$ \\
7 & $4,9,1$ & $4,9,18,1$ & $4,9,18,27,1$ \\
\hline
\end{tabular}




\subsection{Effect of layers on training of neural networks}

Results from Table 5 show that the neural network with 2 hidden layers achieved the lowest percentage of the absolute error. The absolute error $=\mid($ predicted Ra value $)-($ measured Ra value $) \mid /($ measured Ra value $)$. Therefore, the networks with one and two hidden layers were used for further comparisons.

\subsection{Effect of activation functions on training of neural networks}

Two different types of activation functions were used to train all the neural networks, so as to check whether the range of the data form 0 to 1 or -1 to 1 would result in the best. The significance of this variation was evaluated using the ANOVA.

According to Table 6, the design factor for the function was found to be smaller than the control limit, which meant neither the tansig nor the logsig function had a significant effect on the optimization of the network. The evaluation also showed that the design factor for the layers and neurons was greater than the control limit. This meant that the number of hidden layers and neurons had a significant influence on the optimization of the neural networks.

From the results in Table 5, the 4,4,8,1 neural network using the tansig activation function was able to achieve the least mean absolute error of $2.11 \%$. Therefore, the tansig activation function was used for the neural network training.

Table 5. Average percentage for absolute error for each trial network

\begin{tabular}{lllllll}
\hline $\begin{array}{l}\text { Trial } \\
\text { Network }\end{array}$ & $\begin{array}{l}\text { 1 Hidden Layer } \\
\text { (Neurons) }\end{array}$ & Tansig (\%) & Logsig (\%) & $\begin{array}{l}\text { 2 Hidden layer } \\
\text { (Neurons) }\end{array}$ & Tansig (\%) & Logsig (\%) \\
\hline 1 & $4,3,1$ & 18.41 & 29.57 & $4,3,6,1$ & 14.93 & 12.57 \\
2 & $4,4,1$ & 38.99 & 19.54 & $4,4,8,1$ & 2.11 & 5.42 \\
3 & $4,5,1$ & 16.07 & 35.40 & $4,5,10,1$ & 11.87 & 24.55 \\
4 & $4,6,1$ & 21.86 & 28.76 & $4,6,12,1$ & 25.11 & 16.01 \\
5 & $4,7,1$ & 20.05 & 18.96 & $4,7,14,1$ & 32.5 & 15.68 \\
6 & $4,8,1$ & 22.41 & 27.63 & $4,8,16,1$ & 13.06 & 12.11 \\
7 & $4,9,1$ & 22.16 & 20.34 & $4,9,18,1$ & 31.10 & 18.72 \\
\hline
\end{tabular}

Table 6. ANOVA table for effect comparison

\begin{tabular}{llllll}
\hline Source of Variation & Sum of Squares & Degrees of Freedom & Mean Square & F0 & Control Limit \\
\hline Layer and Neuron & 365.33 & 1 & 365.33 & 4.89 & 4.26 \\
Function & 2.34 & 1 & 2.34 & 0.03 & 4.26 \\
Joint Effect & 86.24 & 1 & 86.24 & 1.15 & 4.26 \\
Error & 1792.86 & 24 & 74.70 & - & - \\
Total & 2246.77 & 27 & - & - & - \\
\hline
\end{tabular}

\subsection{Effects of output and input variables on the training of neural networks}

The comparison of how the input variables affect the optimization of neural networks is shown in Table 7 . The table shows that using 4 inputs to train the neural network led to a less absolute error than using either 3 or 5 inputs. The number of data sets from the inputs would affect the training accuracy, because some of the data sets could obstruct the ability of the network to learn the relations of the inputs and outputs.

Table 7. Average percentage of absolute errors for different inputs

\begin{tabular}{llll}
\hline Inputs & Output & Neural Network & Abs Error (\%) \\
\hline Feed Force, Radial Force, Axial Force & Surface roughness & $3,6,1$ & 29.87 \\
Feed Force, Radial Force, Axial Force, Vibration & Surface roughness & $4,8,1$ & 22.41 \\
$\begin{array}{l}\text { Feed Force, Radial Force, Axial Force, } \\
\text { Vibration, Sound }\end{array}$ & Surface roughness & $5,10,1$ & 38.54 \\
\hline
\end{tabular}

\subsection{Optimization of neural networks}

After training all neural networks that were generated, the 4,4,8,1 neural network was the best, as it was able to achieve the least average percentage of the absolute error for the prediction of surface roughness of milled surfaces. According to Table 8 , the maximum and minimum percentages of the absolute error were $6.98 \%$ and $0.11 \%$ respectively, and the average percentage of the absolute error was $2.11 \%$. The 4,4,8,1 network could predict the roughness heights of milled surfaces accurately. Since the testing data were always different from the training data, the small percentage of the absolute error signified that the neural network had learnt the relationship between the inputs and the output. 
Table 8. Average percentage for absolute error on all the 20 sets of testing data

\begin{tabular}{|c|c|c|c|c|c|c|c|}
\hline Readings & $\begin{array}{l}\text { Feed } \\
(\mathrm{N})\end{array}$ & $\begin{array}{l}\text { Radial } \\
(\mathrm{N})\end{array}$ & $\begin{array}{l}\text { Axial } \\
(\mathrm{N})\end{array}$ & $\begin{array}{l}\text { Vibration } \\
(\mathrm{dBV})\end{array}$ & $\begin{array}{l}\text { Actual Ra } \\
(\mu \mathrm{m})\end{array}$ & $\begin{array}{l}\text { Predicted Ra } \\
(\mu \mathrm{m})\end{array}$ & $\begin{array}{l}\text { Abs Error } \\
(\%)\end{array}$ \\
\hline 1 & 387.5 & 491 & 615.5 & -42.19 & 0.4845 & 0.4708 & 2.82 \\
\hline 2 & 402.5 & 540.2 & 634.1 & -43.05 & 0.4899 & 0.4852 & 0.95 \\
\hline 3 & 417.5 & 589.4 & 652.7 & -43.73 & 0.4953 & 0.4903 & 1.00 \\
\hline 4 & 432.5 & 638.6 & 671.3 & -44.83 & 0.5007 & 0.4857 & 2.99 \\
\hline 5 & 447.5 & 687.8 & 689.9 & -45.92 & 0.5061 & 0.4905 & 3.08 \\
\hline 6 & 462.5 & 737 & 708.5 & -58.26 & 0.5115 & 0.5145 & 0.58 \\
\hline 7 & 477.5 & 786.2 & 727.1 & -60.13 & 0.5169 & 0.5495 & 6.30 \\
\hline 8 & 492.5 & 835.4 & 745.7 & -61.26 & 0.64 & 0.6547 & 2.29 \\
\hline 9 & 507.5 & 884.6 & 764.3 & -62.3 & 0.784 & 0.7525 & 4.01 \\
\hline 10 & 522.5 & 933.8 & 782.9 & -63.32 & 0.928 & 0.9085 & 2.10 \\
\hline 11 & 537.5 & 983 & 801.5 & -63.88 & 1.072 & 0.9971 & 6.98 \\
\hline 12 & 552.5 & 1032.2 & 820.1 & -64.84 & 1.216 & 1.2145 & 0.12 \\
\hline 13 & 567.5 & 1081.4 & 838.7 & -65.86 & 1.36 & 1.3584 & 0.11 \\
\hline 14 & 582.5 & 1130.6 & 857.3 & -67.03 & 1.54 & 1.5005 & 2.56 \\
\hline 15 & 597.5 & 1179.8 & 875.9 & -68.11 & 1.567 & 1.5761 & 0.58 \\
\hline 16 & 612.5 & 1229 & 894.5 & -68.32 & 1.594 & 1.5607 & 2.08 \\
\hline 17 & 627.5 & 1278.2 & 913.1 & -69.03 & 1.621 & 1.6139 & 0.43 \\
\hline 18 & 642.5 & 1327.4 & 931.7 & -69.54 & 1.648 & 1.6437 & 0.26 \\
\hline 19 & 657.5 & 1376.6 & 950.3 & -70.54 & 1.675 & 1.6873 & 0.73 \\
\hline 20 & 672.5 & 1425.8 & 968.9 & -72.34 & 1.702 & 1.7404 & 2.25 \\
\hline \multicolumn{7}{|c|}{ Mean percentage for absolute error: } & 2.11 \\
\hline
\end{tabular}

Figure 2 shows that the 4,4,8,1 neural network could learn the nonlinear complex relations of the output and input readings, as also shown in Table 8 . Thus, it was capable of providing a trend accurately for product quality prediction and tool condition monitoring. It can also reduce the machining time and machining costs.

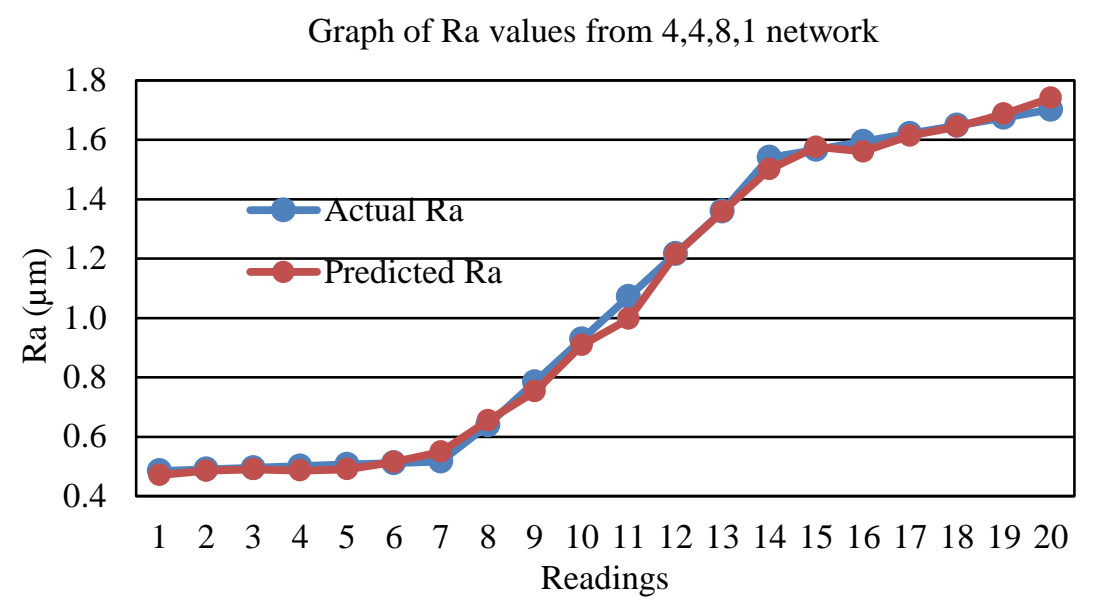

Figure 2. The 4,4,8,1 neural network could learn the nonlinear complex relations of the output and input readings

\subsection{Limitation of the approach}

The network had to be trained with training data sets that would enable it to predict the output according to the relationship that it had learnt, as illustrated in Table 9. Using the testing data sets that were out of the range of the training data sets resulted in inaccurate predictions and the results could never be reliable. This illustrates the requirements for training data sets for accurate prediction of milled surfaces.

Table 9. Predictions of Ra using 3 over ranged testing data

\begin{tabular}{llllll}
\hline Feed $(\mathrm{N})$ & Radial $(\mathrm{N})$ & Axial $(\mathrm{N})$ & Vibration $(\mathrm{dBV})$ & Actual Ra $(\mu \mathrm{m})$ & Predicted Ra $(\mu \mathrm{m})$ \\
\hline 707.5 & 1508.2 & 1003.1 & -77.54 & 1.75 & 0.378 \\
710.0 & 1506.7 & 1005.9 & -78.95 & 1.81 & -11.540 \\
712.4 & 1514.7 & 1022.3 & -83.24 & 1.83 & 1.06 \\
\hline
\end{tabular}




\section{Conclusions}

Cutting forces can be used to predict product quality and monitor tool conditions. As cutting forces in the feed, radial and axial directions increase, surface roughness also increases, which indicates the degradation of product quality and the decrease of the tool life. In addition, the surface roughness increases when the magnitude of the vibration increases. When the milling parameters are fixed to be constant, increased surface roughness is a sign of increased tool wear. Therefore, the roughness can be used to determine the tool condition.

With the aid of neural networks, the data sets collected are used to explore the ability to predict product quality and to monitor tool conditions. The contributions of different parameters of neural networks, which can influence the accuracy of predicting product quality and tool conditions, are analyzed and optimized.

A 4,4,8,1 neural network, with 2 hidden layers using the tansig function, is able to predict surface roughness output data sets by training with the cutting force and vibration input data sets. The minimum error of the prediction of surface roughness is $0.11 \%$, the average error is $2.11 \%$, and the maximum error is $6.98 \%$. The prediction of surface roughness of milled surfaces is successful, which is very important for the product quality prediction and tool condition monitoring.

\section{References}

[1] Guang Y, Zhong ZW, Ding Z. Optimization of tool change timing in a nut forming process using genetic algorithms. Journal of Intelligent Manufacturing. 2004;15(5):693-699.

[2] Zhou JH, Pang CK, Zhong ZW, Lewis FL. Tool wear monitoring using acoustic emissions by dominantfeature identification. IEEE Transactions on Instrumentation and Measurement. 2011;60(2):547-559.

[3] Fu P, Hope AD. Intelligent classification of cutting tool wear states. In: International Symposium on Neural Networks 2006. Springer, Berlin, Heidelberg. 2006. p. 964-969.

[4] Mulc T, Udiljak T, Čuš F, Milfelner M. Monitoring cutting-tool wear using signals from the control system. Journal of Mechanical Engineering. 2004;50(12):568-579.

[5] Dimla DE. Sensor signals for tool-wear monitoring in metal cutting operations - a review of methods. International Journal of Machine Tools and Manufacture. 2000;40(8):1073-1098.

[6] Oguamanam DC, Raafat H, Taboun SM. A machine vision system for wear monitoring and breakage detection of single-point cutting tools. Computers \& Industrial Engineering. 1994;26(3):575-598.

[7] Barakat M, El Badaoui M, Guillet F. Hard competitive growing neural network for the diagnosis of small bearing faults. Mechanical Systems and Signal Processing. 2013;37(1-2):276-292.

[8] Li H, Wang J. Assessment of cutting forces in high-speed milling of Inconel 718 considering the dynamic effects. Proceedings of the Institution of Mechanical Engineers, Part B: Journal of Engineering Manufacture. 2013;227(11):1581-1595.

[9] Liu TI, Jolley B, Yang CH. On-line detection and measurements of tool wear for precision boring of titanium components. Proceedings of the Institution of Mechanical Engineers, Part B: Journal of Engineering Manufacture. 2016;230(7):1331-1342.

[10] Liu TI, Song SD, Liu G, Wu Z. Online monitoring and measurements of tool wear for precision turning of stainless steel parts. The International Journal of Advanced Manufacturing Technology. 2013;65(9-12):13971407.

[11] Worden K, Staszewski WJ, Hensman JJ. Natural computing for mechanical systems research: A tutorial overview. Mechanical Systems and Signal Processing. 2011;25(1):4-111.

[12] Guo YB, Ammula SC. Real-time acoustic emission monitoring for surface damage in hard machining. International Journal of Machine Tools and Manufacture. 2005;45(14):1622-1627.

[13] Mantle AL, Aspinwall DK. Surface integrity of a high speed milled gamma titanium aluminide. Journal of Materials Processing Technology. 2001;118(1-3):143-150.

[14] Cho S, Binsaeid S, Asfour S. Design of multisensor fusion-based tool condition monitoring system in end milling. The International Journal of Advanced Manufacturing Technology. 2010;46(5-8):681-694.

[15] Benardos PG, Vosniakos GC. Prediction of surface roughness in CNC face milling using neural networks and Taguchi's design of experiments. Robotics and Computer-Integrated Manufacturing. 2002;18(5-6):343354.

[16] Dimla Jr DE, Lister PM, Leighton NJ. Neural network solutions to the tool condition monitoring problem in metal cutting - a critical review of methods. International Journal of Machine Tools and Manufacture. 1997;37(9):1219-1241.

[17] Dornfeld DA, DeVries MF. Neural network sensor fusion for tool condition monitoring. CIRP Annals. 1990;39(1):101-105.

[18] Zhong ZW, Khoo LP, Han ST. Prediction of surface roughness of turned surfaces using neural networks. The International Journal of Advanced Manufacturing Technology. 2006;28(7-8):688-693. 
[19] Tansel IN, Arkan TT, Bao WY, Mahendrakar N, Shisler B, Smith D, McCool M. Tool wear estimation in micro-machining.: Part I: tool usage-cutting force relationship. International Journal of Machine Tools and Manufacture. 2000;40(4):599-608.

[20] Zhong ZW, Khoo LP, Han ST. Neural-network predicting of surface finish or cutting parameters for carbide and diamond turning processes. Materials and Manufacturing Processes. 2007;23(1):92-97.

[21] Bouzgou H, Benoudjit N. Multiple architecture system for wind speed prediction. Applied Energy. 2011;88(7):2463-2471.

[22] Lee JH, Lee SJ. One-step-ahead prediction of flank wear using cutting force. International Journal of Machine Tools and Manufacture. 1999;39(11):1747-1760.

[23] Ng JJ. Monitoring of tool conditions for machining of aerospace materials. Report of Nanyang Technological University, Singapore.2014.

[24] Zhou JH, Pang CK, Lewis FL, Zhong ZW. Intelligent diagnosis and prognosis of tool wear using dominant feature identification. IEEE Transactions on Industrial Informatics. 2009;5(4):454-464.

[25] Patterson DW. Artificial neural networks: theory and applications. Prentice Hall PTR; 1998.

[26] Hagan MT, Demuth HB, Beale M. Neural network design. Boston : PWS Publishing Co.; 1996.

(C) 2019 by the author(s). This work is licensed under a Creative Commons Attribution 4.0 International License (http://creativecommons.org/licenses/by/4.0/). Authors retain copyright of their work, with first publication rights granted to Tech Reviews Ltd. 